\title{
Kaspar Podgorski
}

\author{
A yellow fluorescent sensor to study the brain, and the joy and pain of climbing.
}

n n a photo on Kaspar Podgorski's blog, he grips a mountainside with a few fingers as he scopes out his next move. This neuroscientist and fellow at Janelia Research Campus is a serious climber. He talks science when climbing with his favorite climbing partner, also a researcher. Science and climbing do not overlap much, but they have some aspects in common. "I love them both very much," he says. "There's a lot of trying to do things that are hard and getting a lot of joy out of failing at things that are hard."

Right now, climbing is not on his schedule, nor the outdoor photography he enjoys. And as of this interview, Janelia labs were closed because of COVID-19. He does computing tasks related to his projects and rows on the Potomac, also to stay fit for his next climb.

$\mathrm{He}$ and his colleagues have developed jYCaMP for two-photon microscopy. It's a yellow, genetically encoded calcium indicator that excites at frequencies between 1,030 and 1,040 nanometers. The team tested jYCaMP, for example, by recording from neurons in the visual cortex in mice. They labeled presynaptic axons coming from the thalamus to the visual cortex and labeled cortical neurons there, too. Recording from this location in sequential experiments would miss plenty, he says. The alternative is one laser beam that excites calcium indicators emitting at different wavelengths. In testing they found jYCaMP "co-excites really well" with red fluorescent sensors.

They have shared jYCaMP with other labs, where it fills spectral gaps or helps in imaging multiple areas of the brain. "I actually I love getting feedback from folks that are using our tools," he says.

Parallelized two-photon microscopes take much excitation power to image many points in the brain simultaneously. "By exciting more points at the same time, we can speed up imaging," he says. This isn't doable with tunable lasers for a reasonable price, but it works with lower cost fiber lasers. His group needed a sensor that worked with wavelengths above 1,000 nanometers. "That's easier said than done," he says.

To get a bright yellow, they used well-known mutations that turn GFPs into YFPs and inserted them into the widely used jGCaMP7. "And we got an indicator that's still green," he says. A different tack with

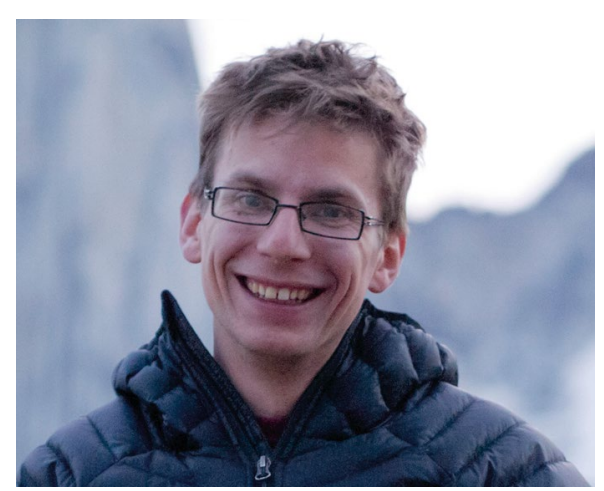

Kaspar Podgorski. Credit: L. Thompson

random mutagenesis and high-throughput screening was needed. And they wanted to detect a mere one-nanometer spectral difference and assess other properties, too. They built a large-field-of-view microscope with two cameras for screening tens of thousands of colonies at a time and that was able to detect very narrow filtered wavelengths of the emission spectrum. They pulled out promising ones and tested aspects such as brightness and response. "It's important in these screens not to be greedy," he says. One property might look great but other properties, such as good expression in the cell, matter too.

Podgorski's overarching goal is to one day "get at how neurons compute," by recording comprehensively the inputs into neurons and understanding how the inputs are transformed into outputs. This involves building microscopes, developing indicators and devising algorithms. He grew up in Toronto and studied physics at the University of Toronto with a biology scholarship. He poked around to combine neuroscience and artificial intelligence. "I sort of moved away from the well-formulated, well-defined problems of physics and math and towards the sort of messy, ill-defined challenges of neurobiology."

He took a class with machine learning pioneer Geoffrey Hinton, who recommended a stint at the Gatsby Computational Neuroscience Unit at University College London. "That was really eye-opening for me," says Podgorski.
Then he moved to Vancouver to spend time climbing and decide what's next. He held two part-time jobs in University of British Columbia labs. One involved software development for visual analytics; the other, neuroscience-based data analysis with Kurt Haas. He joined the Haas lab as a PhD student.

Podgorski was excited about the people he met at a conference at Janelia. An inquiry about a postdoctoral fellowship turned into an invitation to apply as a fellow, a junior 'lab head' post, that he landed in 2015. "My whole life changed at that point," he says.

Podgorski "is one of the smartest and funniest people I know," says Haas. "He has a unique ability to absorb and apply everything effortlessly." He has improved multiple different technologies to advance fast neural imaging. "Podgorski approaches all tasks with the same vigor, whether climbing and setting new routes on mountain walls or overcoming research hurdles in the lab." Haas sought to record all the synaptic input and action potential output activity of a neuron in an intact and awake brain to get closer to understanding, among other aspects, how information sculpts neuronal morphology.

\section{"My whole life changed at that point."}

"Kaspar had no experience in designing and building microscopes, but within a short time constructed an ultra-fast random-access two-photon microscope, complete with driver and analysis software," he says. This was likely the first microscope to fully image sensory-evoked activity throughout a neuron in an awake brain. "After he left my lab to take his fellow position at Janelia, it has taken the combined skills of four new hires in my lab to be able to carry on his work."

\section{Vivien Marx}

Published online: 18 June 2020

https://doi.org/10.1038/s41592-020-0887-8

Reference

Mohr, M. A. et al. jYCaMP: an optimized calcium indicator for two-photon imaging at fiber laser wavelengths. Nat. Methods https://doi.org/10.1038/ s41592-020-0835-7 (2020). 